\title{
Non-Debye Enhancements in the Mie Scattering of Light from a Single Water Droplet
}

James A. Lock

Cleveland State University, j.lock@csuohio.edu

Judith R. Woodruff

Follow this and additional works at: https://engagedscholarship.csuohio.edu/sciphysics_facpub

Part of the Physics Commons

How does access to this work benefit you? Let us know!

\section{Publisher's Statement}

This paper was published in Applied Optics and is made available as an electronic reprint with the permission of OSA. The paper can be found at the following URL on the OSA website: http://www.opticsinfobase.org/ao/abstract.cfm?URI=ao-28-3-523. Systematic or multiple reproduction or distribution to multiple locations via electronic or other means is prohibited and is subject to penalties under law.

\section{Original Citation}

Lock, James A. and Judith R. Woodruff. "Non-Debye Enhancements in the Mie Scattering of Light from a Single Water Droplet." Applied Optics 28 (1989): 523-529.

\section{Repository Citation}

Lock, James A. and Woodruff, Judith R., "Non-Debye Enhancements in the Mie Scattering of Light from a Single Water Droplet" (1989). Physics Faculty Publications. 35.

https://engagedscholarship.csuohio.edu/sciphysics_facpub/35

This Article is brought to you for free and open access by the Physics Department at EngagedScholarship@CSU. It has been accepted for inclusion in Physics Faculty Publications by an authorized administrator of EngagedScholarship@CSU. For more information, please contact library.es@csuohio.edu. 


\title{
Non-Debye enhancements in the Mie scattering of light from a single water droplet
}

\author{
James A. Lock and Judith R. Woodruff
}

\begin{abstract}
The glare spots usually seen on a single water droplet which has been illuminated by a plane wave are produced by geometrical rays which correspond to the different terms of the Debye series expansion of the Mie scattered field. Recently other glare spot enhancements have been predicted which correspond to scattering resonances coupling to the orbiting rays associated with high-order geometrical rainbows. We observed the non-Debye enhancement of the eleventh-order rainbow glare spot at an observation angle of $90^{\circ}$ on a $3.5-\mathrm{mm}$ water droplet illuminated by polarized $\mathrm{He}-\mathrm{Ne}$ laser light.
\end{abstract}

\section{Introduction}

When a plane wave of light illuminates a spherical water droplet, an observer reasonably close to the droplet and focusing his eyes on it sees several spots of glare at various locations on its surface. At certain observation angles known as the rainbow angles, pairs of these glare spots merge and take on color. When the observer is distant from the thousands of droplets present in a rain shower, the usual atmospheric rainbow is the combined effect of the coloration of each of the droplets which is at the observer's rainbow angle. ${ }^{1}$ The spots of glare seen on a single droplet are produced by geometrical light rays making a given number of internal reflections within the droplet before exiting it. These multiply internally reflecting light rays arise from the different-order terms of the Debye series expansion of the Mie scattered electric field. ${ }^{2-3}$ For a spherical drop, the behavior of the Debye term glare spots as a function of the observation angle is both theoretically understood ${ }^{4,5}$ and experimentally confirmed. 4 Recently, numerical calculations of the Fourier transform of the Mie electric field showed additional sharp enhancements in the spatial frequency spectrum, which could not be totally attributed to any single term of the Debye series. ${ }^{6}$ Rather, these additional enhancements are produced by scattering reso-

When this work was done both authors were with Cleveland State University, Physics Department, Cleveland, Ohio 44115; J. R. Woodruff is now with Kent State University, Physics Department, Kent, Ohio 44242.

Received 23 May 1988.

0003-6935/89/030523-07\$02.00/0.

(C) 1989 Optical Society of America. nances coupling to the orbiting rays associated with high-order geometrical rainbows. This paper describes an experiment whose purpose was to observe such a non-Debye glare spot on a single water droplet.

Scattering experiments employing a single water droplet are of two general types. In the first type of experiment, the Mie intensity is measured as a function of the observation angle by employing either a very small receiving optics aperture without a focusing lens ${ }^{7}$ or a larger aperture with a lens focused on infinity. ${ }^{8}$ In these experiments, rainbows appear as strong narrow enhancements in the Mie intensity. They are accompanied on one side by the oscillatory supernumerary intensity and on the other side by a uniformly dark region known as Alexander's band. In the second type of experiment, the square of the Fourier transform of the Mie electric field is measured by employing a receiving optics lens focused on the droplet. 4,8 When the observer is at one of the rainbow angles, a rainbow appears as a colored glare spot which we denote by $R^{(m)}$ at a certain location on the droplet surface, where $m$ is the number of internal reflections that the light rays producing the rainbow make within the droplet. When the observer moves away from the rainbow angle in one direction, the coloration leaves the glare spot and it breaks into the two supernumerary glare spots $R_{a}^{(m)}$ and $R_{b}^{(m)}$. When the observer moves away in the other direction, the glare spot vanishes. The two kinds of experiment are sensitive to different types of information about the scattering. The first experiment accurately measures the oscillatory structure of the angular dependence of the intensity. But it poorly resolves high-order rainbows hiding in the supernumerary structure of lower-order rainbows. Since overlapping signals with different periodicities become separated in the Fourier transform domain, the second 
experiment permits a clean observation of high-order rainbows. The experiment described here is of this second type.

\section{Theoretical Considerations and Previous Experiments}

The pendant water droplets employed in the experiments of Ref. 4 and 7 were not spherical. Rather their shape may be modeled by the prolate spheroid

$$
\frac{x^{2}}{a^{2}}+\frac{y^{2}}{b^{2}}+\frac{z^{2}}{a^{2}}=1
$$

with the $y$ axis vertical, $a / b<1$, and with the incident light rays parallel to the $z$ axis. Water droplets suspended from a hypodermic syringe with its point ground flat ${ }^{9-11}$ have aspect ratios in the $0.84 \lesssim a / b \lesssim$ 0.91 range. The intensity of the light scattered from a spheroid may be exactly calculated in terms of a partial wave series. ${ }^{12}$ However, the computational methods thus far employed in the calculation become numerically unstable ${ }^{13}$ for size parameters $[(2 \pi a) / \lambda] \gtrsim 30$, while the size parameter corresponding to a $3.5-\mathrm{mm}$ diam droplet is $\sim 17,000$. As a result, the light scattered from a pendant water droplet is calculated from a combination of Mie scattering sphere results and geometrical ray tracing.

For the prolate spheroid geometry of a pendant drop and the oblate spheroid geometry of a falling raindrop, the cross section of the droplet in the horizontal $y=0$ midplane is circular and the droplet surface is normal to it. Thus light rays incident on the droplet in the midplane remain in that plane during and after the scattering. Mathematically the scattered intensity in the midplane is well described by Mie scattering from a sphere. However, light rays which enter the droplet slightly above or below the midplane leave the initial plane of incidence at subsequent internal reflections for $a / b \neq 1$ and follow skew paths within the droplet. For certain droplet aspect ratios, certain of these skew rays exit the droplet parallel to the midplane and interfere with the midplane rays producing a higher-order catastrophe caustic at the rainbow location.

In the first type of scattering experiment described in the previous section, the entire higher-order caustic structure is observed when the scattered light intensity is measured in two angular dimensions as is the case with the photographs of Ref. 8 . If the scattered intensity is only measured in one angular dimension, i.e., along the caustic axis as is the case when using a polar nephelometer, the skew ray caustic manifests itself as an amplification of the Mie rainbow intensity. In the second type of scattering experiment, the skew ray caustic appears as two additional glare spots on the droplet. These additional glare spots lie above and below the midplane glare spots or appear as an arc of light along the edge of the droplet extending upward and downward from the rainbow glare spot. The effect of skew rays on rainbow formation has been observed $^{8}$ and analyzed $8,14,15$ for the $m=1$ rainbow for oblate spheroidal water droplets.

Since the present scattering experiment is performed on a pendant water droplet, care must be taken to insure that the droplet aspect ratio is such that skew ray caustic formation does not occur. Otherwise any rainbow enhancement due to scattering resonanceorbiting ray coupling that might be present would be masked by the catastrophe caustic produced by the interference of horizontally emerging skew rays with the horizontal midplane rays. For the prolate spheroidal geometry, skew rays making only one internal reflection within the droplet cannot exit it parallel to the midplane. However, skew rays can emerge parallel to the midplane after $m \geq 2$ internal reflections. In the vicinity of the $m \geq 2$ rainbows, the midplane rays that form the $R_{a}^{(m)}$ and $R_{b}^{(m)}$ glare spots enter the droplet near its outer edge. The paths of the skew rays which enter the droplet near its outer edge but above or below the midplane were calculated for $2 \leq m \leq 11$ internal reflections. In this calculation, the incident light rays were parallel to the $z$ axis and intersected the droplet of Eq. (1) at

$$
\begin{aligned}
& x=a \sin \theta_{i}=a\left(1-\frac{\delta_{0}^{2}}{2}\right), \\
& y=\epsilon_{0} b,
\end{aligned}
$$

where $\epsilon_{0} \ll 1, \delta_{0} \ll 1$, and $\epsilon_{0}^{2} \lesssim \delta_{0}^{2}-\left(\delta_{0}^{4} / 4\right)$. To first order in $\epsilon_{0}$, the aspect ratios of the droplets whose skew rays of Eq. (2) emerge horizontally after $m$ internal reflections are given in Table I. To first order in $\delta_{0}$ and $\epsilon_{0}$, the deviation angle of the skew ray in the horizontal plane $\Delta^{(m)}$ is found to be

$$
\Delta^{(m)}=\Delta_{0}^{(m)}+(2 m+2)\left[\delta_{0}-\left(\delta_{0}^{2}-\epsilon_{0}^{2}\right)^{1 / 2}\right],
$$

where $\Delta_{0}^{(m)}$ is the deviation of the corresponding midplane ray which enters the droplet at

$$
\begin{aligned}
& x=a \sin \theta_{i}, \\
& y=0, \\
& z=-a \cos \theta_{i} .
\end{aligned}
$$

For pendant water droplets with aspect ratios of the

\begin{tabular}{cccc}
$\begin{array}{c}\text { Table I. Aspect Ratlos of Pendant Droplets for which Skew Rays Enter } \\
\text { Above or Below the Midplane near its Outer Edge, Make } \boldsymbol{m} \text { Internal } \\
\text { Reflectlons Within the Droplet, and Exit Parallel to the Midplane }\end{array}$ \\
\hline$m$ & $a / b$ & $m$ & $a / b$ \\
\hline & & 1 & - \\
2 & 0.7576 & 3 & 0.5799 \\
4 & 0.8907 & 5 & 0.7576 \\
& 0.4683 & & 0.3922 \\
6 & 0.9448 & 7 & 0.8418 \\
& 0.6575 & & 0.5799 \\
& 0.3372 & & 0.2956 \\
8 & 0.9740 & 9 & 0.8907 \\
& 0.7576 & & 0.6880 \\
& 0.5183 & & 0.4683 \\
& 0.2631 & & 0.2371 \\
10 & 0.9921 & 11 & 0.9224 \\
& 0.8193 & & 0.7576 \\
& 0.6295 & & 0.5799 \\
& 0.4270 & & 0.3922 \\
& 0.2156 & & 0.1977 \\
\hline
\end{tabular}


order of $a / b \sim 0.9$, it is seen from Table I that skew rays affect the even-order rainbows more severely than they do the odd-order ones.

Using a polar nephelometer to measure the scattered intensity in the droplet midplane, Sassen ${ }^{7}$ conjectured that skew rays contributed to the following features of the scattered intensity that he observed: (1) the ratio of the intensity of the second-order rainbow to the intensity of the first-order rainbow $R^{(2)} / R^{(1)}$ increased as the horizontal droplet radius $a$ increased; (2) for large $a$, there was a strong enhancement of the $R^{(3)}+$ $R^{(4)}$ rainbow; (3) $R^{(6)}$ was surprisingly strong for both large and small droplets; (4) no $R^{(5)}$ or $R^{(7)}$ rainbow structure was observed; and (5) enhanced depolarized scattering was observed in the angular interval $80^{\circ} \lesssim \theta_{0}$ $\lesssim 100^{\circ}$ for large droplets, and for different drops the enhanced depolarized scattering was seldom encountered in the same angular positions. The results of Table I and Eq. (3) demonstrate the importance of horizontally emerging skew rays in producing these features. Since the aspect ratio decreases as the droplet diameter increases and since $a / b \sim 0.89$ for $a=1.4$ $\mathrm{mm}$, features (1)-(3) may be explained in terms of enhanced $R^{(2)}, R^{(4)}$, and $R^{(6)}$ scattering along the hyperbolic umbilic caustic axis for $\epsilon_{0} \ll \delta_{0}$ when the aspect ratio of the droplet is near the values given in Table I. This enhancement is more difficult to observe in $R^{(8)}$, $R^{(10)}$, and $R^{(12)}$, since these rainbows appear near the forward scattering or backscattering directions. Feature (4) is consistent with the fact that the aspect ratio of the droplet must be quite small to enhance an oddorder rainbow rather than an adjacent $m$ even-order rainbow. When modeled as a sphere, the intensity of the light scattered from the droplet midplane possesses a broad relative minimum for $90^{\circ} \lesssim \theta_{0} \lesssim 110^{\circ}$. In the vicinity of the fourth-order rainbow, $\delta_{0}=0.18$ and $\Delta_{0}^{(4)}=402.74^{\circ}$. With these values, feature (5) may be explained in terms of weakly enhanced $R^{(4)}$ scattering for $\epsilon_{0} \sim 0.8 \delta_{0}$. At the appropriate aspect ratio, these skew rays exit the droplet parallel to the midplane near $\theta_{0} \approx 90^{\circ}$, thus filling in the relative minimum of the Mie sphere intensity in that interval. For very large water droplets, weakly enhanced $R^{(2)}$ scattering may also play a role for $\epsilon_{0} \sim 0.8 \delta_{0}$ since near the second rainbow $\delta_{0}=0.31$ and $\Delta_{0}^{(2)}=230.40^{\circ}$. The variability of the angular position of feature (5) may well reflect a variability of the aspect ratio from droplet to droplet.

If a pendant droplet can remain near spherical in shape, $a / b \sim 1$ and higher-order caustic formation should not occur in conjunction with the odd-order rainbows. To produce near spherical droplets, water droplets were formed on the $2.5-\mathrm{mm}$ diam base of a glass pipet rather than on the $0.75-\mathrm{mm}$ diam hypodermic syringe as had been the case in Refs. 4, 7, and 9 . The resulting aspect ratios of the droplets suspended in this way were $a / b \sim 0.94$. To minimize the possibility of producing higher-order rainbow caustics due to skew rays, it was decided to examine the non-Debye enhancement of odd-order rainbows only.
Because the different-order Debye term glare spots are produced by stationary phase portions of the partial wave sum, they are equally visible in both temporally coherent and incoherent illumination. The nonDebye enhancements are produced by a different mechanism, namely, by having many terms of the internal reflection series for the resonant partial wave, corresponding to different path lengths, in constructive interference with each other. For a droplet whose diameter is a few millimeters, the incident plane wave must be temporally coherent over a length of a few millimeters as well to insure that the phase of each of the terms of the internal reflection series has its proper value. This coherence length restriction requires that laser light be employed in the search for non-Debye enhancements. They will not appear in temporally incoherent light such as from an incandescent light bulb. In the present experiment, we employed the light from a $\mathrm{He}-\mathrm{Ne}$ laser for which

$$
\lambda=0.6328 \mu \mathrm{m},
$$

and the index of refraction of water is

$$
n=1.3317 \text {. }
$$

In the visual observations reported by Walker, ${ }^{4,9}$ he described a surprisingly bright $R^{(11)}$ rainbow when illuminating the droplet with the temporally incoherent light from a slide projector lamp. His enhanced eleventh-order rainbow turns out to be produced by the skew ray hyperbolic umbilic caustic. The droplet used in his observations had an aspect ratio of $a / b \sim 0.87$, and photographs of his eleventh-order rainbow show it to consist of a glare spot in the droplet midplane and two additional glare spots $0.4 \mathrm{~mm}$ above and below the midplane. ${ }^{11}$ Although these three glare spots are clearly resolvable on photographs of the droplet, they are too close together and too bright compared to their dark surroundings to be resolved by visual observations. Visually they give the impression of a single bright glare spot.

From numerical calculations it was found that, although the non-Debye enhancements occur in conjunction with all the high-order rainbows, the strength of the enhancement slowly increases as $m$ increases. This is probably a result of the $R_{c}^{(m)}$ orbiting ray relative minimum of the phase of the scattered amplitude becoming stronger and more well defined as $m$ increases and thus providing a more stable structure for resonant partial waves to couple onto. Of the oddorder rainbows for which skew ray caustics should not be problematical for a near spherical drop, it was decided to search for the non-Debye enhancement with $m$ as large as possible, i.e., for $R^{(11)}$ at $\theta_{0}=90^{\circ}$. The $m$ $=13$ rainbow was not considered since this rainbow glare spot overlaps the much brighter transmitted ray glare spot. Although Table I shows that aspect ratios in the vicinity of $a / b \sim 0.92$ will produce the skew ray caustic which can obscure the non-Debye enhancement of $R^{(11)}$, the wide pipet base produces droplets with aspect ratios substantially larger than those used by Walker for which the skew ray caustic was observed. 
Thus skew ray caustics should be able to be avoided for the geometry of the present experiment.

We define the 1 polarization state of the normally incident plane wave as the geometry where the incident electric field is parallel to the pendant droplet symmetry axis and the 2 polarization state as the geometry where the normally incident electric field is perpendicular to it. ${ }^{16}$ The various-order Debye term rainbow glare spots are known to be strongly polarized in the 1 state. ${ }^{4,7}$ Near $90^{\circ}$, the direct reflection glare spot is also strongly polarized in the 1 state. Since the $R^{(11)}$ non-Debye glare spot at $90^{\circ}$ is expected to occur on the droplet surface near the much brighter direct reflection glare spot $G$ and the two-internal-reflection glare spot $R_{a}^{(2)}$, it might well be obscured by them unless these brighter glare spots can be suppressed. Mie calculations for the spherical geometry show that at $\theta_{0} \approx 90^{\circ}$, the non-Debye glare spot is nearly unpolarized, but the direct reflection glare spot is 18 times less intense in the 2 state than in the 1 state and the twointernal-reflection glare spot is 30 times less intense in the 2 state. Thus to minimize the brightness of the unwanted nearby glare spots when searching for the non-Debye enhancement, we used only the 2 polarization state of the incident laser light.

For the parameters

$$
\begin{aligned}
\theta_{0} & =90.0^{\circ}, \\
a & \approx 1.75 \mathrm{~mm}, \\
\theta_{w} & =4.8^{\circ},
\end{aligned}
$$

where $\theta_{w}$ is the range of observation angles subtended by the focusing lens, and for the numerical values given in eqs. (5) and (6) the square of the Fourier transform of the Mie electric field convolved with the observer's aperture function was calculated as a function of spatial frequency for the 2 polarization. Graphs of such Fourier transform spectra when the scattering resonance-orbiting ray coupling is at its maximum and minimum strength are shown in Figs. 1 and 2, respectively. Similarly, for

$$
\theta_{0}=84.0^{\circ}
$$

away from the eleventh-order rainbow location, the square of the Fourier transform of the Mie electric field convolved with the observer's aperture function was also calculated as a function of spatial frequency for the 2 polarization. This spectrum was found to be independent of $a$ and is shown in Fig. 3. The area under each of the glare spot enhancements in these figures compared with the area under the direct reflection glare spot at $\theta_{0}=90^{\circ}$ is given in Table II. In this table, the entry $R^{(11)}\left(90^{\circ}\right) / G\left(90^{\circ}\right)$ is the area under the enhancement produced by the geometric eleven-internal-reflection portion of the scattering amplitude alone. Since Figs. 1-3 describe the intensity profile along a 1-D slice through actual 2-D glare spots, comparisons of the power present in actual glare spots require that the squares of the ratios given in Table II be used.

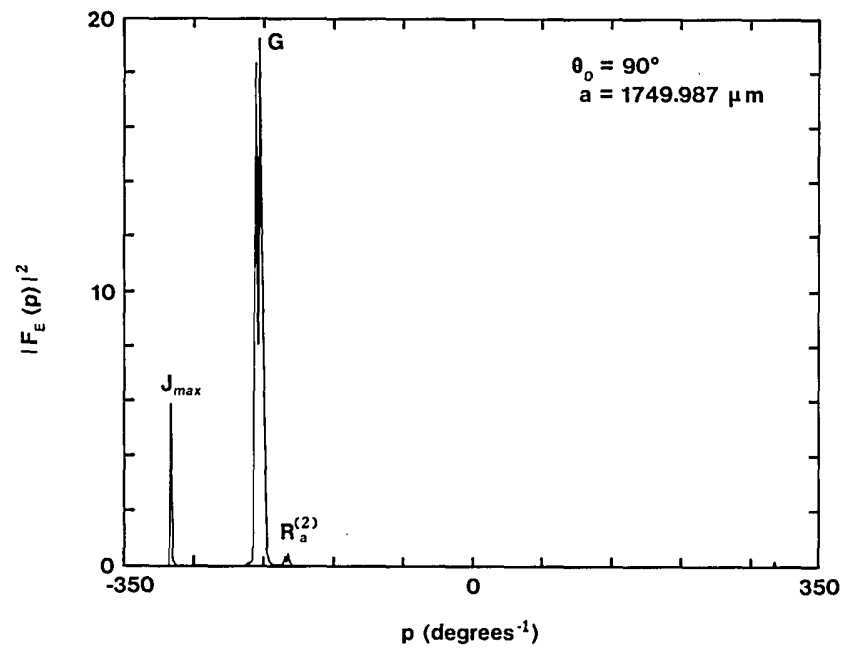

Fig. 1. Square of the Fourier transform of the Mie scattered field convolved with the observer's aperture function for the 2 polarization state and plotted as a function of the spatial frequency $p$ for $\theta_{0}=$ $90.0^{\circ}$ and $a=1749.987 \mu \mathrm{m}$. $G$ is the direct reflection glare spot, $R_{a}^{(2)}$ is one of the two-internal-reflection glare spots, and $J$ is the nonDebye enhancement of the $R^{(11)}$ glare spot at maximum strength. The edges of the droplet are at $p= \pm 303.27 \mathrm{deg}^{-1}$.

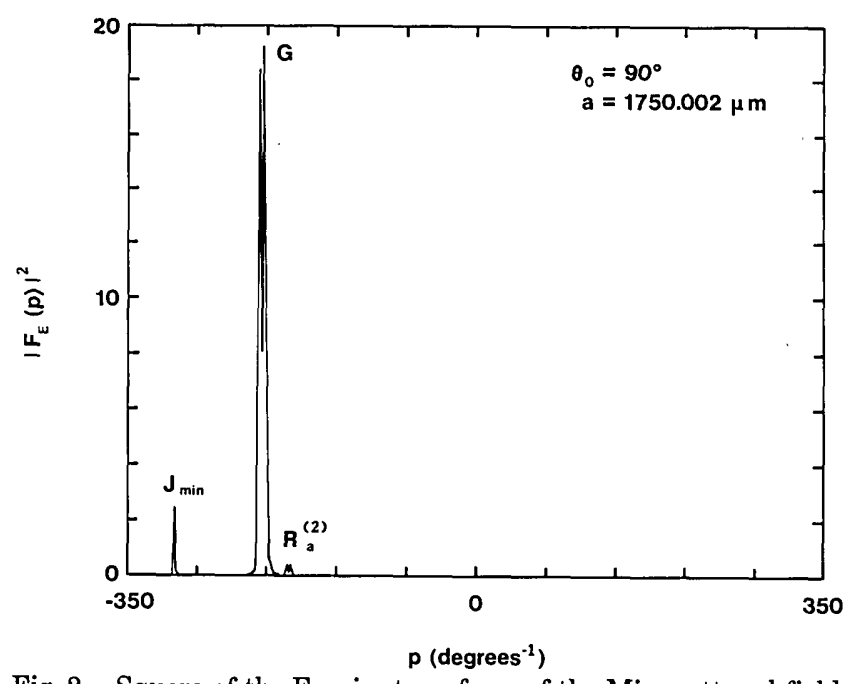

Fig. 2. Square of the Fourier transform of the Mie scattered field convolved with the observer's aperture function for the 2 polarization state and plotted as a function of the spatial frequency $p$ for $\theta_{0}=$ $90.0^{\circ}$ and $a=1750.002 \mu \mathrm{m}$. $G$ and $R_{a}^{(2)}$ are as in Fig. 1 and $J$ is the non-Debye enhancement of the $R^{(11)}$ glare spot at minimum strength.

Since a strong resonance is produced for about every $0.01-\mu \mathrm{m}$ increase or decrease in $a$, the natural evaporation rate of the droplet ${ }^{10}$ causes $\sim 33$ resonances/s to couple one after the other to high-order rainbow orbiting rays. As a result, the intensity of the $J$ glare spot oscillates back and forth between the maximum and minimum conditions such as those shown in Figs. 1 and 2. The resulting time averaged area under the nonDebye glare spot during a long exposure period and assuming a uniform evaporation rate is given by the entry in Table II labeled $J_{\text {ave }}\left(90^{\circ}\right) / G\left(90^{\circ}\right)$. The time averaged non-Debye glare spot area of Table II is $22.4 \%$ 


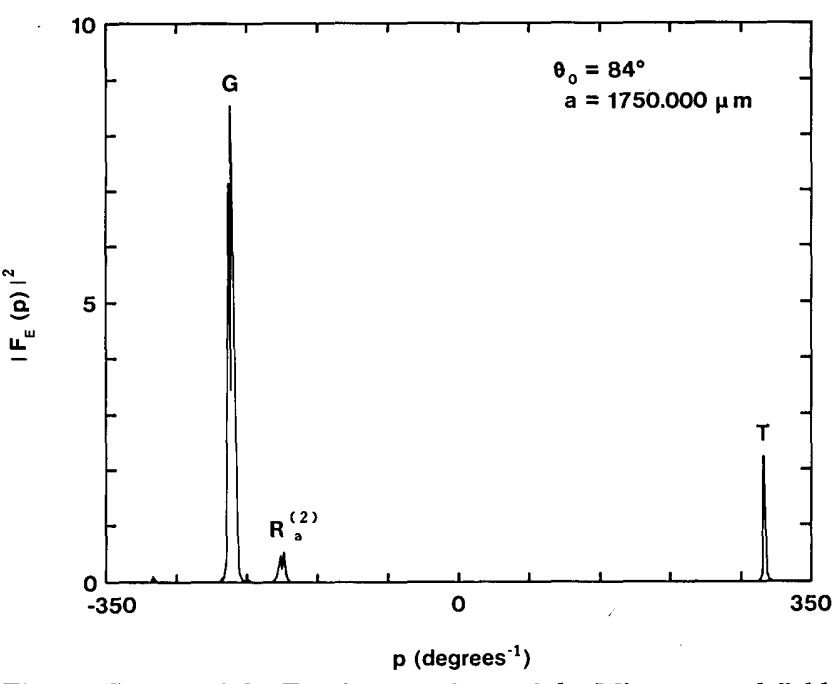

Fig. 3. Square of the Fourier transform of the Mie scattered field convolved with the observer's aperture function for the 2 polarization state and plotted as a function of the spatial frequency $p$ for $\theta_{0}=$ $84.0^{\circ}$ and $a=1750.000 \mu \mathrm{m}$. $G$ and $R_{a}^{(2)}$ are as in Fig. 1 and $T$ is the transmitted ray glare spot.

higher than that obtained from the geometrical eleventh-order rainbow alone.

\section{Experimental Procedure}

A block diagram of the experimental setup is shown in Fig. 4. The output beam of a model 159 SpectraPhysics 10-mW. He-Ne laser was spatially filtered. It was then expanded to a beam diameter of $75 \mathrm{~mm}$ and collimated by a second lens. The expanded beam was aligned so that there was no beam drift in either the horizontal or vertical directions. The beam was then passed through a polarizing filter oriented in the 2 polarization state as described in the previous section. A pipet with a $2.5-\mathrm{mm}$ base was filled with normal tap water that had been allowed to stand for one month in a closed container to degas. The pipet was vertically mounted on a lab stand in a cardboard box with one open side. A water droplet with a horizontal diameter of $\sim 3.5 \mathrm{~mm}$ was formed and suspended from the lower end of the pipet. The evaporation rate of the droplet was measured to be comparable with that of the control drop of Ref. 10. Both the pipet and inside surfaces of the box had been spray-painted flat black to reduce spurious reflections within the box. Enclosing the droplet within the box prevented its illumination by light sources other than the laser. The box further

Table II. Ratlos of the Areas Under the Glare Spot Enhancements of Figs. $1-3$

\begin{tabular}{lccc}
\multicolumn{4}{c}{$1-3$} \\
\hline & $\theta_{0}=84.0^{\circ}$ & $\theta_{0}=84.13^{\circ}$ & $\theta_{0}=90.0^{\circ}$ \\
\cline { 2 - 4 }$G\left(\theta_{0}\right) / G\left(90^{\circ}\right)$ & 0.4196 & 0.4305 & 1.0000 \\
$R_{a}^{(2)}\left(\theta_{0}\right) / G\left(90^{\circ}\right)$ & 0.0323 & 0.0333 & 0.0215 \\
$T\left(\theta_{0}\right) / G\left(90^{\circ}\right)$ & 0.0360 & 0.0328 & - \\
$R^{11}\left(\theta_{0}\right) / G\left(90^{\circ}\right)$ & - & - & 0.0424 \\
$J_{\min }\left(\theta_{0}\right) / G\left(90^{\circ}\right)$ & - & - & 0.0289 \\
$J_{\max }\left(\theta_{0}\right) / G\left(90^{\circ}\right)$ & - & - & 0.0761 \\
$J_{\text {ave }}\left(\theta_{0}\right) / G\left(90^{\circ}\right)$ & - & - & 0.0519 \\
\hline
\end{tabular}

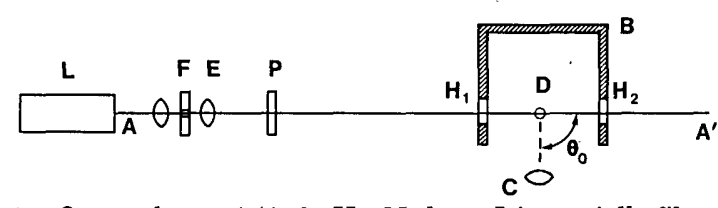

Fig. 4. Output beam $A A^{\prime}$ of a $\mathrm{He}-\mathrm{Ne}$ laser $L$ is spatially filtered by the pinhole filter $F$, expanded and collimated by the second lens $E$, and polarized by the polarizing filter $P$. It passes through entrance and exit holes $H_{1}$ and $H_{2}$ of the box $B$. Light scattered from the water droplet $D$ in the direction of the observation angle $\theta_{0}$ is incident on the camera lens $C$.

protected the droplet from vibrations caused by air currents in the room.

After a horizontal traverse of $7 \mathrm{~m}$, the central portion of the expanded laser beam entered the box through a hole cut into one side and exited through an oversized hole cut into the opposite side. To avoid illuminating the pipet, the bottom of the pipet was lined up with the top of the beam entrance hole. The illuminated droplet was photographed on an Ektachrome 400 color slide film with a tripod-mounted Canon AE1 SLR camera equipped with an antireflection coated Tamron model $28 \mathrm{~A}$ zoom lens. The camera was positioned at the open side of the box enclosure $10 \mathrm{~cm}$ from the droplet at observation angles of $\theta_{0}=90 \pm 1^{\circ}$ and $\theta_{0}=84 \pm 1^{\circ}$. The camera $f$-stop was adjusted so that the effective lens diameter subtended an angle of $\theta_{w}=4.8^{\circ}$. The center of the camera's focusing ring was aligned with the midplane of the pendant drop to ensure that the camera received the light scattered by the droplet midplane. Once the camera was positioned, aligned, and focused on the droplet, the room lights were extinguished and time exposure photographs of from 20 to $120 \mathrm{~s}$ of the illuminated droplet were taken. After the slides were developed, they were placed in a slide projector and focused onto a viewing screen $13 \mathrm{~m}$ away. The viewing screen was then replaced by an HAD$1100 \mathrm{~A}$ photodiode/operational amplifier, ${ }^{17}$ and the power in each glare spot was measured. In determining the glare spot power that exposed the color slide film, the film nonlinearity and photodiode nonlinearity were measured and compensated for.

\section{Results and Discussion}

Photographs of a $3.5-\mathrm{mm}$ diam illuminated pendant water droplet taken at observation angles of $\theta_{0}=90 \pm$ $1^{\circ}$ and $\theta_{0}=84 \pm 1^{\circ}$ are shown in Figs. 5 and 6 , respectively. The photograph of Fig. 5 qualitatively exhibits the glare spot structure predicted in Figs. 1 and 2. The glare spots $G$ and $R_{a}^{(2)}$, which are separated by $0.16 \mathrm{~mm}$ on the droplet, are clearly resolvable. A glare spot is also observed at the location of the $J$, and no additional glare spots attributable to skew rays appear above or below it. Thus we are assured that none of the intensity of the $J$ is due to the hyperbolic umbilic caustic which would have likely occurred had the droplet aspect ratio been smaller. Similarly we believe it to be unlikely that any of the $J$ glare spot intensity is an artifact created by spurious reflections within the camera itself. As mentioned earlier, the Tamron zoom 


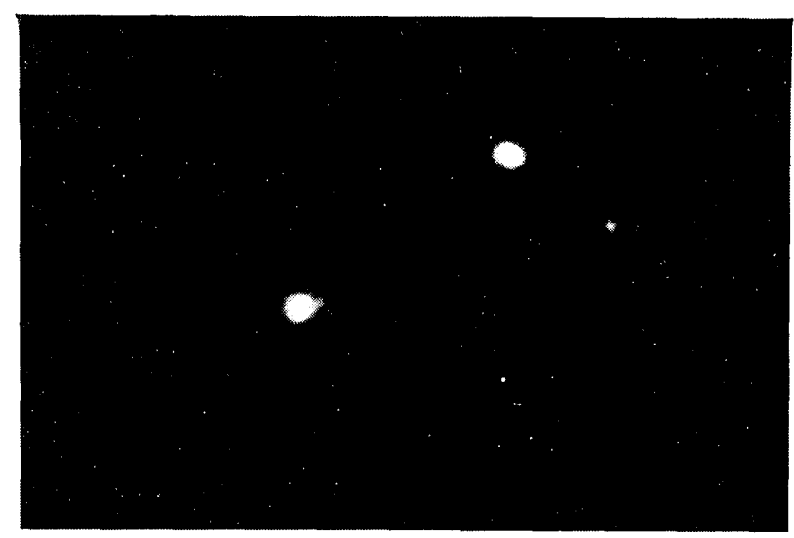

Fig. 5. Glare spots on the droplet midplane at an observation angle of $\theta_{0}=90 \pm 1^{\circ}$. The droplet diameter was $3.5 \mathrm{~mm}$, and the exposure time was $120 \mathrm{~s}$.

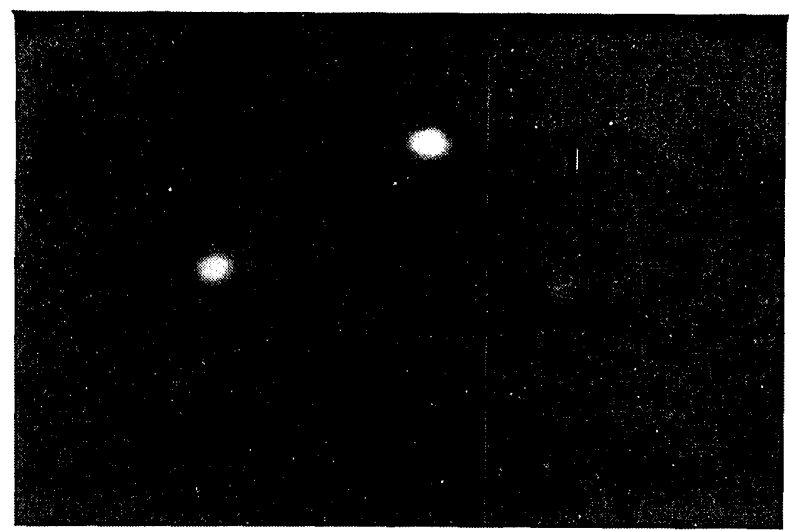

Fig. 6. Glare spots on the droplet midplane at an observation angle of $\theta_{0}=84 \pm 1^{\circ}$. The droplet diameter was $3.5 \mathrm{~mm}$, and the exposure time was $60 \mathrm{~s}$.

lens employed is antireflection coated and its mount has flat back surfaces. Spurious reflections often take the form of caustics, diffraction halos, or diffraction spikes. ${ }^{18}$ No such structures were seen at any location on any of the photographs. For the longest exposures and without the polarizing filter in place, diffraction spikes began to form only on the brightest glare spot $G$. Any veiling glare that might have been present and would have covered the glare spots would also have been present in the background and was subtracted out in the analysis of the photodiode data. The photograph of Fig. 6 qualitatively exhibits the glare spot structure predicted in Fig. 3 . The glare spots $G, R_{a}^{(2)}$, and $T$ are clearly visible. The glare spots at the top of Figs. 5 and 6 are associated with the connection of the neck of the pendant drop to the base of the pipet. These glare spots had a similar appearance in each of the forty-five photographs.

Measurements of the power in each of the glare spots produced three significant results. First, in the neighborhood of $\theta_{0}=84^{\circ}$, the ratio $G\left(\theta_{0}\right) / G\left(90^{\circ}\right)$ of Table II is a rapidly varying function of $\theta_{0}$. Thus the power measured in the $G$ glare spot can be used as a check on the angular positioning of the camera. Assuming that the ratio of the powers in the glare spots is proportional to the square of $G\left(\theta_{0}\right) / G\left(90^{\circ}\right)$ of Table II, the experimental power ratio of $0.185 \pm 0.006$ was found to be within $5.2 \%$ of the result predicted for the camera positioned at $\theta_{0}=84.0^{\circ}$. If the experimental value of the power ratio for the direct reflection glare spots at $\theta_{0}$ and $90^{\circ}$ were instead used to determine independently the observation angle $\theta_{0}$ of the camera, we obtain $\theta_{0}=$ $84.13 \pm 0.09^{\circ}$. The numerically calculated area under the glare spots $G, R_{a}^{(2)}$, and $T$ for $\theta_{0}=84.13^{\circ}$ is given in Table II.

The second result concerns the comparative strengths of $R_{a}^{(2)}$ and $T$. For the index of refraction of Eq. (6), the scattering angle of the critically refracted transmitted ray is $\theta_{0}=82.66^{\circ}$. For scattering angles beyond this critical value, the strength of the transmitted ray rapidly dies off mirroring the Fock transition of the corresponding scattering amplitude. For a nominal scattering angle of $\theta_{0}=84.0^{\circ}$ and with the angular width of the camera lens being given by Eq. (9), the camera receives light from scattering angles in the $81.6^{\circ} \leq \theta \leq 86.4^{\circ}$ range. As a result, the power in the $T$ glare spot is a rapidly decreasing function of $\theta_{0}$ as is the ratio $T\left(\theta_{0}\right) / R_{a}^{(2)}\left(\theta_{0}\right)$. This ratio, along with the direct reflection glare spot ratio previously described; serves as a sensitive test of the accuracy of the recording and measurement of the glare spot powers. For $\theta_{0}=84.13^{\circ}$ as deduced from the previous glare spot ratio, the square of the ratio $T\left(\theta_{0}\right) / R_{a}^{(2)}\left(\theta_{0}\right)$ from Table II is 0.97 . The experimentally determined ratio of the power in the $T$ glare spot in Fig. 6 to the power in the $R_{a}^{(2)}$ glare spot was found to be $1.01 \pm 0.07$ in agreement with the theoretical value. Alternatively, if the experimental value of this ratio were used to obtain an independent evaluation of $\theta_{0}$, we obtain $\theta_{0}=84.11 \pm 0.03^{\circ}$.

The third result concerns the strength of the $J$ glare spot. As shown in Sec. II, if scattering resonances couple the orbiting ray associated with the eleventhorder rainbow and if the evaporation rate of the drop is constant, the power in the $J$ glare spot should be 1.50 times as large as it would have been for the geometrical eleventh-order rainbow alone. The measured power in the $J$ glare spot was compared with the measured powers in the $R_{a}^{(2)}\left(84.13^{\circ}\right)$ and $T\left(84.13^{\circ}\right)$ glare spots, since the power in the $R_{a}^{(2)}\left(90^{\circ}\right)$ glare spot could not be reliably determined due to its proximity to $G\left(90^{\circ}\right)$. Using these results, the measured power in the $J\left(90^{\circ}\right)$ glare spot was $1.36 \pm 0.15$ of the expected eleventhorder rainbow power. This result provides evidence for our having observed the scattering enhancement caused by the coupling of a sequence of scattering resonances to the eleventh-order rainbow orbiting ray.

The experiments of Ref. 10 show that the evaporation of a pendant droplet is a complicated phenomenon. Although the time averaged evaporation rate is uniform, there are short-lived fluctuations in the evaporation rate. When the droplet radius is such that a scattering resonance occurs, the resonance stores a large amount of electromagnetic field energy within the droplet. If this resonant energy storage temporarily increases the evaporation rate, one might find that the evaporation rate is slightly larger on-resonance and 
slightly smaller off-resonance. If this were the case, the time averaged value of $J_{\text {ave }}\left(90^{\circ}\right) / G\left(90^{\circ}\right)$ would be slightly lower than the value given in Table II. Our determination of the measured power in the $J\left(90^{\circ}\right)$ glare spot is consistent with such a lowering of the $J_{\text {ave }}\left(90^{\circ}\right) / G\left(90^{\circ}\right)$ ratio. The uniformity or nonuniformity of the evaporation rate could be tested if the oscillations in the $J\left(90^{\circ}\right)$ glare spot power were measured as a function of time. Experiments which measured resonant fluctuations in the radiation pressure have been performed on smaller droplets. ${ }^{19}$ Thus the time resolved observation of non-Debye scattering enhancements could serve as a test of various evaporation models.

The authors thank Jearl D. Walker for making his rainbow slides available to us. We also thank Robert C. Anderson of NASA Lewis Research Center and Edward A. Hovenac of Sverdrup Technology, Inc. for the loan of the photodiode and its supporting electronics.

\section{References}

1. D. S. Falk, D. R. Brill, and D. G. Stork, Seeing the Light (Harper \& Row, New York, 1986), Sec. 2.6B.

2. H. M. Nussenzveig, "High-Frequency Scattering by a Transparent Sphere. I: Direct Reflection and Transmission," J. Math. Phys. 10, 82 (1969).

3. H. M. Nussenzveig, "High-Frequency Scattering by a Transparent Sphere. II: Theory of the Rainbow and the Glory," J. Math. Phys. 10, 125 (1969).

4. J. D. Walker, "Multiple Rainbows from Single Drops of Water and Other Liquids," Am J. Phys. 44, 421 (1976).
5. J. A. Lock, "Theory of the Observations Made of High-Order Rainbows from a Single Water Droplet," Appl. Opt. 26, 5291 (1987).

6. J. A. Lock, "Cooperative Effects Among Partial Waves in Mie Scattering," J. Opt. Soc. Am. A (in press) (1989).

7. K. Sassen, "Angular Scattering and Rainbow Formation in Pendant Drops," J. Opt. Soc. Am. 69, 1083 (1979).

8. P.L. Marston and E. H. Trinh, "Hyperbolic Umbilic Diffraction Catastrophe and Rainbow Scattering from Spheroidal Drops," Nature, London 312, 529 (1984).

9. J. D. Walker, "How to Create and Observe a Dozen Rainbows in a Single Drop of Water," Sci. Am. 237, 138 (July 1977), Fig. 1.

10. K. Sassen, "Infrared (10.6- $\mu \mathrm{m})$ Radiation Induced Evaporation of Large Water Drops," J. Opt. Soc. Am. 71, 887 (1981), Fig. 3 (left).

11. J. D. Walker, Cleveland State University, Physics Dept.; private communication.

12. S. Asano and G. Yamamoto, "Light Scattering by a Spheriodal Particle," Appl. Opt. 14, 29 (1975).

13. S. Asano, "Light Scattering Properties of Spheroidal Particles," Appl. Opt. 18, 712 (1979).

14. J. F. Nye, "Rainbow Scattering from Spheroidal Drops-an Explanation of the Hyperbolic Umbilic Foci," Nature London 312, 531 (1984).

15. P. L. Marston, "Cusp Diffraction Catastrophe from Spheroids: Generalized Rainbow and Inverse Scattering," Opt. Lett. 10, 588 (1985).

16. This is the convention employed in H. C. Van de Hulst, Light Scattering by Small Particles (Dover, New York, 1981), Sec. 15.12; M. Kerker, The Scattering of Light and Other Electromagnetic Radiation (Academic, New York, 1969), Sec. 6.1.4; and C. F. Bohren and D. R. Huffman, Absorption and Scattering of Light by Small Particles (Wiley, New York, 1983), Sec. 8.4.

17. Manufactured by EG\&G Electro-Optics, 35 Congress St., Salem, MA 01970.

18. See, for example, Astronomy 14, 99 (May 1986).

19. A. Ashkin and J. M. Dziedzic, "Observation of Resonances in the Radiation Pressure on Dielectric Spheres," Phys. Rev. Lett. 38, 1351 (1977).
Course Title:

Course Topics:

Contact:

Course Length:

Dates/Locations:

\section{OPTICAL COMPONENT \& SYSTEMS}

Optical Components and Systems is a "hardware-oriented" course that deals with the function, design, selection, testing and use of specific devices that are currently found in complex optical and electro-optical systems. The content of the course is organized around specific optical phenomena; after the technical principles are discussed, devices are described, along with their characteristics, specifications, practical tolerances, and limitations. The practical aspects of the course are emphasized through individual and group sessions, case studies, and a tour through a major optical fabrication facility, examples and demonstrations of a variety of optical/electro-optical devices and testing techniques for verifying specifications and performance requirements.

\author{
ENGINEERING TECHNOLOGY INSTITUTE \\ P.O. Box 8859 \\ Waco, TX 76714-8859 \\ 1-800-367-4238
}

5 Days

Cost: $\$ 960.00$

May 8-12, 1989

Dallas, TX 